\section{Mehr Wissen - mehr Lebensqualität für Prostatakrebspatienten}

\begin{abstract}
- Mit dem Histrelin-Jahresimplantat Vantas ${ }^{\circledR}$ bietet die Firma Orion Pharma Patienten mit fortgeschrittenem Prostatakarzinom eine effektive Hormon-Suppressionstherapie. Die Galenik des Implantats ermöglicht eine kontinuierliche Wirkstofffreisetzung über zwölf Monate. Begleitend zu dieser Therapie hat Orion Pharma mit "Vantas ${ }^{\circledR}$ : Das Serviceprogramm" ein umfassendes Paket zum Thema Prostatakrebs erstellt, das Prostatakarzinompatienten ausführlich über Krankheit, Diagnose und Therapieoptionen informiert.

„Vantas ${ }^{\oplus}$ : Das Serviceprogramm" liefert in Form von sieben Broschüren viele nützliche Informationen zu verschiedenen Aspekten der Erkrankung, Therapien und begleitende, die Lebensqualität steigernde Behandlungen. Ärzte können ihren Patienten somit umfassende Informationen an die Hand geben, die den Betroffenen und Angehörigen helfen, den Herausforderungen besser zu
\end{abstract}

begegnen. Die Kompendien sind von ausgewiesenen Experten patientengerecht und besonders anschaulich aufbereitet. Sie bieten unter dem Motto "Mehr Wissen - mehr Lebensqualität" neben Basisinformationen zum Prostatakrebs Wissenswertes rund um das Leben mit Krebs und behandeln die Schwerpunktthemen Lebenszufriedenheit, mentale Stärke, Bewegung, Ernährung, Partnerschaft/ Freundeskreis und Sexualität. Integrierte Fragebögen ermöglichen den Patienten eine genaue Reflexion der einzelnen Themen und sollen dazu beitragen, dass das neu erworbene Wissen im Alltag umgesetzt wird.

Bestellmöglichkeit des Serviceprogramms sowie weitere Informationen auf www. vantas-pca.de oder unter der kostenlosen Servicehotline von Orion Pharma 0800-82 68271.

Nach Informationen von

Orion Pharma, Hamburg
Wissensquiz zum unkontrollierten Harndrang

In der interaktiven Fortbildung „Toviaz ${ }^{\circledast}$ Wissensquiz" von Pfizer wird Ihnen das Arzneimittel Toviaz ${ }^{\circledast}$ (Fesoterodin) zur Behandlung des unkontrollierten Harndrangs vorgestellt. Im Frage- und Antwortspiel können Sie Ihr Wissen zum unkontrollierten Harndrang testen.

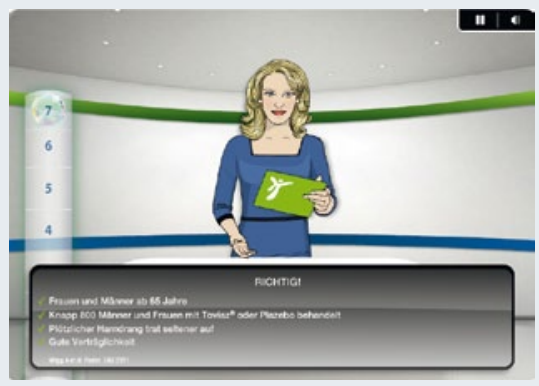

Gleichzeitig erfahren Sie, welche Vorteile Fesoterodin bei der Behandlung dieser Krankheit bereit hält. Haben Sie Lust bekommen, sich den Fragen zu stellen? Dann werden Sie Kandidat: www.toviaz-interaktiv.de.

Nach Informationen von Pfizer, Berlin

\section{Website zu chronischen Zystitiden}

Als wahrscheinliche Ursache chronischer Zystitiden gilt ein Defekt der äußersten Blasenschutzschicht (Glykosaminoglykan-, GAG-Schicht). Die intravesikale Instillation mit Gepan ${ }^{\oplus}$ instill versorgt die GAGSchicht mit ihrem Baustein Chondroitinsulfat und behebt den Defekt. Davon profitieren Patienten mit verschiedenen Formen der chronischen Zystitis: Symptome wie imperativer Harndrang, hohe Miktionsfrequenz und Schmerzen werden deutlich gelindert. Auf www.gepan-instill. de beantworten Experten die wichtigsten Fragen zu chronischen Zystitiden - Ursachen, Symptome und Behandlungsmethoden. Videos erklären den Wirkmechanismus von Gepan ${ }^{\circledast}$ instill und zeigen, wie eine zielgerichtete Beckenboden-Gymnastik die Instillationstherapie unterstützt.

Die GAG-Ersatztherapie wird derzeit nicht von den gesetzlichen Krankenkassen erstattet. Um die Patienten wirtschaftlich zu entlasten, gewährt Pohl-Boskamp einen Preisnachlassauf die jeweiligen Packungsgrößen. Auf www.gepan-instill.de können sich Betroffene diesen Preisvorteil sichern.

Nach Informationen von

Pohl-Boskamp, Hohenlockstedt 